\title{
557.
}

\section{ON CERTAIN CONSTRUCTIONS FOR BICIRCULAR QUARTICS.}

[From the Proceedings of the London Mathematical Society, vol. v. (1873-1874), pp. 29-31. Read March 12, 1874.]

I CALL to mind that if $F, G$ are any two points and $F^{\prime}, G^{\prime}$ their antipoints; then the circle on the diameter $F G$ and that on the diameter $F^{\prime \prime} G^{\prime}$ are concentric orthotomics, viz. they have the same centre, and the sum of the squared radii is $=0$. Moreover, if the circles $B, B^{\prime}$ are concentric orthotomics, and the circle $A$ is orthotomic to $B$, then it is a bisector of $B^{\prime}$, viz. it cuts $B^{\prime}$ at the extremities of a diameter of $B^{\prime}$; and $B^{\prime}$ is then said to be a bifid circle in regard to $A$.

Given two real circles, these have an axial orthotomic, the circle, centre on the line of centres at its intersection with the radical axis, which cuts at right angles the given circles; viz. this axial orthotomic is real if the circles have no real intersection; but if the intersections are real, then the axial orthotomic is a pure imaginary, and instead thereof we may consider its concentric orthotomic, viz. this is the axial bifid of the two circles, or circle having its centre on the line of centres at the intersection thereof with the radical axis or common chord of the two circles, and having this common chord for its diameter.

If one of the circles is a pure imaginary, then we have still an axial orthotomic; viz. the pure imaginary circle is replaced by the concentric orthotomic; and the axial orthotomic is a bisector of the substituted circle; and so if each of the circles is a pure imaginary, then we have still an axial orthotomic, viz. each circle is replaced by the concentric orthotomic, and the axial orthotomic is a bisector of the substituted circles. And in either case the axial orthotomic of the original circles (one or each of them pure imaginary) is real; viz. this is given either as the axial bisector of one real circle and orthotomic of another real circle; or as the axial bisector of two circles, from which the reality thereof easily appears. Or we may verify it thus: Suppose 
that the two circles are $(x-\alpha)^{2}+y^{2}=\beta^{2},\left(x-\alpha^{\prime}\right)^{2}+y^{2}=\beta^{\prime 2}$, and their axial orthotomic $(x-m)^{2}+y^{2}=k^{2}$, then we have $(m-\alpha)^{2}=\beta^{2}+k^{2},\left(m-\alpha^{\prime}\right)^{2}=\beta^{\prime 2}+k^{2}$; subtracting, it appears that $m$ is real; and then if either $\beta^{2}$ or $\beta^{\prime 2}$ is negative, the equation containing this quantity shows that $k^{2}$ is positive; viz. the circle $(x-m)^{2}+y^{2}=k^{2}$ is real.

The above remarks have an obvious application to the theory of bicircular quartics; viz. a bicircular quartic is the envelope of a variable circle, having its centre on a conic, and orthotomic to a circle: it may be that this circle is a pure imaginary. We then replace it by the concentric orthotomic, and say that the curve is the envelope of a variable circle having its centre on a conic and bisecting a circle. We have thus a real form for cases which originally present themselves under an imaginary form.

\section{The Bicircular Quartic with given vertices.}

First, if the vertices are real; let the vertices taken in order be $F, G, H, K$.

First construction: $O n F G$ as diameter describe a circle, and on $H K$ as diameter a circle; on the line terminated by the two centres (as transverse or conjugate axis) describe a conic $\Theta_{1}$, and describe the axial orthotomic circle $\Sigma_{1}$ of the two circles (viz. the centre of $\Sigma_{1}$ is on the axis of symmetry at its intersection with the radical axis of the two circles); then the curve is the envelope of a variable circle having its centre on $\Theta_{1}$ and orthotomic to $\Sigma_{1}$.

Second construction: On $F H$ as diameter describe a circle, and on $G K$ as diameter a circle. On the line terminated by the two centres (as transverse or conjugate axis) describe a conic $\Theta_{2}$, and describe the axial bifid circle $\Sigma_{2}^{\prime}$ of the two circles (viz. the centre of $\Sigma_{2}^{\prime}$ is on the axis of symmetry at its intersection with the radical axis or common chord of the two circles, and its diameter is this common chord); then the curve is the envelope of a variable circle having its centre on $\Theta_{2}$ and bisecting $\Sigma_{2}^{\prime}$.

Third construction: On $F K$ as diameter describe a circle, and on $G H$ as diameter a circle; and then, as in the first construction, à conic $\Theta_{3}$ and a circle $\Sigma_{3}$; the curve is the envelope of a variable circle having its centre on $\Theta_{3}$ and orthotomic to $\Sigma_{3}$.

Observe that in the three constructions the conics have always the same centre; and if the three conics are taken with the same foci, then the three constructions give one and the same bicircular quartic. The first and third constructions form a pair, and there is no reason for selecting one of them in preference to the other; but the second construction is unique; it is on this account natural to make use of it in discussing the series of curves with the given vertices.

In the particular case where the points $F, G$ and $H, K$ are situate symmetrically on opposite sides of a centre $O\left(O F^{\prime}=O K, O G=O H\right)$, then in the third construction the centres each coincide with $O$, or the axis of the conic vanishes; hence the construction fails: the first and second constructions hold good, and in each of them the 
conic and circle are concentric. The curve is in this case quadrantal: having, besides the original axis of symmetry, another axis of symmetry through 0 , at right angles thereto.

Secondly, if the vertices are two real, two imaginary, say $f, g=\alpha \pm \beta \iota ; h$, $k$, we modify the first or third construction; viz. if $F^{\prime}, G^{\prime}$ are the antipoints of $F, G$; then on $F^{\prime} G^{\prime}$ as diameter describe a circle, and on $H K$ as diameter a circle. On the line terminated by the two centres (as transverse or conjugate axis) describe a conic $\Theta_{1}$, and describe the axial bisector-orthotomic circle $\Sigma_{1}$ of the two circles; viz. this is the circle (centre on the axis of symmetry) which bisects the circle $F^{\prime} G^{\prime}$, and cuts at right angles the circle $H K$; then the curve is the envelope of the variable circle having its centre on $\Theta_{1}$ and orthotomic to $\Sigma_{1}$.

Thirdly, if the vertices are all imaginary, say $f, g=\alpha \pm \beta \iota ; h, k=\gamma \pm \delta \iota$, we modify the first or third construction. Take $F^{\prime}, G^{\prime}$ the antipoints of $F, G$, and $H^{\prime}, K^{\prime}$ the antipoints of $H, K$; then on $F^{\prime} G^{\prime}$ as diameter describe a circle, and on $H^{\prime} K^{\prime}$ as diameter a circle; on the line terminated by the two centres (as transverse or conjugate axis) describe a conic $\Theta$, and describe the axial bisector-circle $\Sigma$ of the two circles (viz. this is a circle, centre on the axis of symmetry, bisecting each of the circles): the curve is the envelope of a variable circle, centre on the conic $\Theta$ and cutting at right angles the circle $\Sigma$. 